\title{
Hopf bifurcation analysis of nonlinear HIV infection model and the effect of delayed immune response with drug therapies
}

\section{Geetha ${ }^{1}$ and S. Balamuralitharan ${ }^{2^{*}}$}

\author{
"Correspondence: \\ balamurs@srmist.edu.in \\ ${ }^{2}$ Department of Mathematics, \\ Faculty of Engineering and \\ Technology, SRM Institute of \\ Science and Technology, \\ Kattankulathur-603 203, \\ Chengalpattu District, Tamil Nadu, \\ India \\ Full list of author information is \\ available at the end of the article
}

\begin{abstract}
A mathematical model of HIV infection with the combination of drug therapy including cytotoxic T-lymphocyte $(C T L)$ and the antibody immune response is examined. The threshold value represented as the basic reproduction ratio $R_{0}$ is derived. This reveals that $R_{0}<1$ is locally asymptotically stable in the viral free steady state, and the infected steady state condition remains locally asymptotically stable with $R_{0}>1$ in the absence of a delay in the immune response. Moreover, the existence of Hopf bifurcation with CTL response delay is demonstrated. The estimation of delay length is used to maintain stability. Numerical simulations are implemented to explain the mathematical results.
\end{abstract}

MSC: 92B05; 34D20; 97M60

Keywords: HIV Delay model; Local Stability; Hopf bifurcation

\section{Introduction}

Human immunodeficiency virus (HIV) is a simple form of virus that affects the human body, and it primarily targets $\mathrm{CD} 4^{+} \mathrm{T}$-cells. Viruses, in general, are the infectious creatures that have no reproductive ability by themselves. They depend on a host for replication to begin. HIV viruses carry a copy of their RNA that needs to first be duplicated into DNA [1]. Furthermore, once the DNA virus has been copied into these host cells, new virus particles must also be assembled at the host cell surface [2]. The maturing for these new particles might occur gradually without impacting that host cell, or it quickly erupts and destroys the host cell $[3,4]$. Antiretroviral therapy (ART) would intend to inhibit that activity for different HIV viral proteins and consequently hinder the viral replication cycle $[5,6]$.The drug based on the inhibitor of reverse transcriptase (RTI) is an obstacle to the reverse transcription of viral DNA and prevents a productive infection of the host cell. Protease inhibitor (PI) drugs aim to suppress protease activity and prevent the development of mature viruses. The combination of reverse transcriptase inhibitor and protease inhibitor is more effective to treat HIV patients.

Mathematical modeling can contribute to the study of antiviral infection treatment and increase knowledge about the virus transmission rate [7, 8]. Numerous models of viral

(c) The Author(s) 2020. This article is licensed under a Creative Commons Attribution 4.0 International License, which permits use, sharing, adaptation, distribution and reproduction in any medium or format, as long as you give appropriate credit to the original author(s) and the source, provide a link to the Creative Commons licence, and indicate if changes were made. The images or other third party material in this article are included in the article's Creative Commons licence, unless indicated otherwise in a credit line to the material. If material is not included in the article's Creative Commons licence and your intended use is not permitted by statutory regulation or exceeds the permitted use, you will need to obtain permission directly from the copyright holder. To view a copy of this licence, visit http://creativecommons.org/licenses/by/4.0/. 
infections are discussed in the literature for HIV dynamics [9]. The immune system CTL plays a vital role in protecting against HIV and this ability prevents the reproduction of HIV [10]. Hence many mathematical models examine viral infection in conjunction with CTL responses [11-13]. To resist viral infection, both CTL immune response and strong antibody neutralizing are necessary for an effective vaccine $[14,15]$.

In [16] Wodarz suggested a model depicting the relationship between CTL and antibody immune responses. The global analysis for this model was provided by Yousfi et al. [17]. Yan and Wang included the intracellular delay in the cell infected model and analyzed the global dynamics effect of the delay [18].

The mathematical study is needed for an integrated view of the dynamics of viruses for delayed models [19-21]. Several models have been suggested with a time lag in triggering the immune reaction when the body is infected with the virus, which is called immunological delay [22-24].

In [25] Dubey et al. described the dynamics of infection by HIV involving available drug therapies, reverse transcriptase inhibitor (RTI), and protease inhibitor (PI) that includes uninfected CD $4^{+}$T-cells $x(t)$, infected cells $y(t)$, free virus $v(t)$, and CTL immune response $C(t)$, and antibody $A(t)$. The non-linear differential equations of the first order are as follows:

$$
\begin{aligned}
& \dot{x}=\lambda+r x\left(1-\frac{x}{x_{m}}\right)-\lambda_{0} x-\beta\left(1-\eta_{r}\right) x v, \\
& \dot{y}=\beta\left(1-\eta_{r}\right) x v-\delta_{0} y-\omega_{1} C y, \\
& \dot{v}=N \delta_{0}\left(1-\eta_{p}\right) y-\delta_{1} v-\omega_{2} A v, \\
& \dot{C}=\alpha_{1} y+\mu_{1} C y-\mu_{10} C, \\
& \dot{A}=\mu_{2} v A-\mu_{20} A,
\end{aligned}
$$

where $\delta_{0}=\delta_{0}^{1}+\frac{\omega_{1} \alpha_{0}}{\mu_{10}}, \alpha_{1}=\alpha_{1}^{1}+\frac{\mu_{1} \alpha_{0}}{\mu_{10}}$.

Here, $\lambda$ is the production level for uninfected $C D 4^{+}$T-cells, $\lambda_{0}$ is an uninfected $C D 4^{+}$ T-cells mortality rate, $r$ is the maximum rate of proliferation, $\beta$ is the infection rate of uninfected cells by the virus, $x_{m}=x_{\max }$ is their carrying capacity, $\eta_{r}$ is the rate of inhibitor reverse transcriptase therapy to destroy infected cells, and $\eta_{p}$ is the rate at which the protease inhibitor therapy blocks the infection, $\delta_{0}^{1}$ is the infected cell mortality rate, $N$ is the number of particles of virus produced by the cell infected, $\delta_{1}$ is the rate of virus removed from the body by natural factors, $\alpha_{0}$ is the rate of produced CTL immune response, $\mu_{10}$ is the deplete rate of CTL. $\alpha_{1}^{1}$ is the stimulated rate of CTL, $\mu_{1}$ is the rate at which CTL interact with the infected cells, $\omega_{1}$ is the level of CTL interaction removed from infected cells. $\mu_{2}$ is the rate at which antibody gets stimulated, $\mu_{20}$ is the deplete rate of antibody due to increase in virus particles. $\omega_{2}$ is the reduced rate of virus with antibody interaction. In this study, we implement a time delay to illustrate the CTL response model. The generation of CTL due to antigenic stimulation requires a period of time $\tau$ and the response to CTL in time $\mathrm{t}$ that depends on an antigen population in the previous time $t-\tau$ for a time lag $\tau>0$. The model suggested by incorporating immune response delay in CTL is 
as follows:

$$
\begin{aligned}
& \dot{x}=\lambda+r x\left(1-\frac{x}{x_{m}}\right)-\lambda_{0} x-\beta\left(1-\eta_{r}\right) x v, \\
& \dot{y}=\beta\left(1-\eta_{r}\right) x v-\delta_{0} y-\omega_{1} C y, \\
& \dot{v}=N \delta_{0}\left(1-\eta_{p}\right) y-\delta_{1} v-\omega_{2} A v, \\
& \dot{C}=\alpha_{1} y+\mu_{1} C(t-\tau) y(t-\tau)-\mu_{10} C, \\
& \dot{A}=\mu_{2} v A-\mu_{20} A .
\end{aligned}
$$

The primary objective is to analyze the impact of immune reaction delay in the dynamics of (2) together with reverse transcriptase inhibitor and protease inhibitor and show the impact on the stability of the delay in the immune response.

We start with some notes that are used in the sequel to our study.

Let $\mathbb{C}=\mathbb{C}\left([-\tau, 0], R_{+}^{5}\right)$ be the Banach space of continuous functions mapping the interval $[-\tau, 0]$ in to $R_{+}^{5}$, where $R_{+}^{5}=(x, y, v, C, A)$.

The initial conditions are given as follows:

$$
\begin{array}{ll}
x(\theta)=\phi_{1}(\theta) \geq 0, & y(\theta)=\phi_{2}(\theta) \geq 0, \quad v(\theta)=\phi_{3}(\theta) \geq 0, \\
C(\theta)=\phi_{4}(\theta) \geq 0, & A(\theta)=\phi_{5}(\theta) \geq 0, \quad \theta \in[-\tau, 0],
\end{array}
$$

where $\phi_{i}(\theta) \in \zeta^{1}$ are smooth functions for all $i=1,2,3,4,5$.

The solution $(x(t), y(t), v(t), C(t), A(t))$ is easy to recognize from the basic theory of differential functions of (2) with the initial conditions as mentioned above which exist for all $t \geq 0$ and are unique [26-28]. It can be shown that this solution exists for all $t>0$ and stays nonnegative. In fact, if $x(0)>0$, then $x(t)>0$ for all $t>0$. The same argument is true for $y, v, C$, and $A$ components. Hence, the interior $R_{+}^{5}$ is invariant for system (2).

The basic reproduction ratio $R_{0}$ of model (2) is defined by

$$
R_{0}=\frac{\beta N\left(1-\eta_{c}\right)}{\delta_{1}} x_{0}
$$

where $\eta_{c}=1-\left(1-\eta_{r}\right)\left(1-\eta_{p}\right)$.

\section{Stability of viral free steady state $\left(I_{0}\right)$}

Theorem 2.1 If $R_{0}<1$ then the viral free steady state $\mathrm{I}_{0}$ is locally asymptotically stable.

Proof The viral free steady state of system (2) is $\mathrm{I}_{0}\left(x_{0}, 0,0,0,0\right)$, where

$$
x_{0}=\frac{x_{m}}{2 r}\left[\left(r-\lambda_{0}\right)+\sqrt{\left(r-\lambda_{0}\right)^{2}+\frac{4 r \lambda}{x_{m}}}\right] \text {. }
$$


The characteristic equation of $I_{0}$ is given by

$$
\left|\begin{array}{ccccc}
-h-\xi & 0 & -\beta_{1} x_{0} & 0 & 0 \\
0 & -\delta_{0}-\xi & \beta_{1} x_{0} & 0 & 0 \\
0 & N_{1} \delta_{0} & -\delta_{1}-\xi & 0 & 0 \\
0 & 0 & 0 & -\mu_{10}-\xi & 0 \\
0 & 0 & 0 & 0 & -\mu_{20}-\xi
\end{array}\right|=0
$$

where

$$
h=\left(\frac{2 r x_{0}}{x_{m}}-\left(r-\lambda_{0}\right)\right)=\sqrt{\left(r-\lambda_{0}\right)^{2}+\frac{4 r \lambda}{c_{m}}}>0, \quad N_{1}=N\left(1-\eta_{P}\right)
$$

and

$$
(\xi+h)\left(\xi+\mu_{20}\right)\left(\xi+\mu_{10}\right)\left[\varepsilon^{2}+\left(\delta_{0}+\delta_{1}\right) \xi+\left(\delta_{0} \delta_{1}-\beta_{1} N_{1} \delta_{0} x_{0}\right)\right]=0 .
$$

From (3) we get $\xi_{1}=-h<0, \xi_{2}=-\mu_{20}<0, \xi_{3}=-\mu_{10}<0$; if $R_{0}<1$ then $\delta_{0} \delta_{1}-$ $\beta_{1} N_{1} \delta_{0} x_{0}>0$.

Thus, the eigenvalues of (3) have negative real parts. Hence the viral free steady state of (2) is locally asymptotically stable.

\section{Stability analysis of infected steady state $\left(I^{*}\right)$ and existence of Hopf \\ bifurcation}

Theorem 3.1 If $R_{0}>1$ then (i) the infected steady state $I^{*}$ of system (2) is locally asymptotically stable for $\tau=0$;

(ii) Time delay $\tau$ crosses the critical value $\tau_{0}$, then system (2) undergoes Hopf bifurcation at the infected steady state $I^{*}$.

Proof The infected steady state $\mathrm{I}^{*}\left(x^{*}, y^{*}, v^{*}, C^{*}, A^{*}\right)$ of (2) is given by

$$
\begin{aligned}
& v^{*}=\frac{\mu_{20}}{\mu_{2}}, y^{*}=\frac{\mu_{10} C^{*}}{\alpha_{1}+\mu_{1} C^{*}}, \quad A^{*}=\frac{N_{1} \delta_{0} \mu_{2} y^{*}-\delta_{1} \mu_{20}}{\mu_{20} \omega_{2}}, \\
& C^{*}=\frac{h_{2}+\sqrt{h_{2}^{2}+4 h_{1} h_{3}}}{2 h_{1}}, \\
& x^{*}=\frac{x_{m}}{2 r}\left[f+\sqrt{f^{2}+\frac{4 r \lambda}{x_{m}}}\right],
\end{aligned}
$$

where

$$
\begin{aligned}
& f=r-\lambda_{0}-\frac{\beta_{1} \mu_{20}}{\mu_{2}}, \quad h_{1}=\mu_{10} \mu_{2} \omega_{1}, \\
& h_{2}=\beta_{1} \mu_{20} \mu_{1} x^{*}-\delta_{0} \mu_{10} \mu_{2}, \quad h_{3}=\beta_{1} \mu_{20} \alpha_{1} x^{*} .
\end{aligned}
$$

The uninfected $\mathrm{CD} 4^{+} \mathrm{T}$ cells $\left(x^{*}\right)$ can achieve a steady state value by resolving the equation

$$
\frac{r}{x_{m}} x^{* 2}+\left(-r+\lambda_{0}+\beta_{1} \frac{\mu_{20}}{\mu_{2}}\right) x^{*}-\lambda=0 .
$$


The immune response $\left(C^{*}\right)$ can obtain a steady state value by solving the following equation:

$$
\omega_{1} C^{*^{2}} \mu_{10} \mu_{2}+C^{*}\left(\delta_{0} \mu_{10} \mu_{2}-\mu_{1} \beta\left(1-\eta_{r}\right) x^{*} \mu_{20}\right)-\beta\left(1-\eta_{r}\right) x^{*} \mu_{20} \alpha_{1}=0 .
$$

The characteristic equation of $I^{*}$ is given by

$$
\begin{array}{|ccccc}
r-\frac{2 r x^{*}}{x_{m}}-\lambda_{0}-\beta_{1} v^{*}-\xi & 0 & -\beta_{1} x^{*} & 0 & 0 \\
\beta_{1} v^{*} & -\left(\delta_{0}+\omega_{1} C^{*}\right)-\xi & \beta_{1} x^{*} & -\omega_{1} y^{*} & 0 \\
0 & N_{1} \delta_{0} & -\left(\delta_{1}+\omega_{2} A^{*}\right)-\xi & 0 & -\omega_{2} v^{*} \\
0 & \mu_{1} C^{*} e^{-\xi \tau}+\alpha_{1} & 0 & \mu_{1} y^{*} e^{-\xi \tau}-\mu_{10}-\xi & 0 \\
0 & 0 & \mu_{2} A^{*} & 0 & \mu_{2} v^{*}-\mu_{20}-\xi
\end{array} \mid
$$

where

$$
\begin{aligned}
& b_{1}=r-\frac{2 r x^{*}}{x_{m}}-\lambda_{0}-\beta_{1} v^{*}, \quad b_{2}=-\beta_{1} x^{*}, \quad b_{3}=\beta_{1} v^{*}, \quad b_{4}=-\left(\delta_{0}+\omega_{1} C^{*}\right), \\
& b_{5}=-\omega_{1} y^{*}, \quad b_{6}=N_{1} \delta_{0}, \quad b_{7}=-\left(\delta_{1}+\omega_{2} A^{*}\right), \quad b_{8}=-\omega_{2} v^{*}, \quad b_{9}=\mu_{1} C^{*}, \\
& b_{10}=\alpha_{1}, \quad b_{11}=\mu_{1} y^{*}, \quad b_{12}=-\mu_{10}, \quad b_{13}=\mu_{2} A^{*}, \quad b_{14}=\mu_{2} v^{*}-\mu_{20} .
\end{aligned}
$$

This is equivalent to the following equation:

$$
\begin{aligned}
\xi^{5}+ & k_{1} \xi^{4}+k_{2} \xi^{3}+k_{3} \xi^{2}+k_{4} \xi+k_{5}+e^{-\xi \tau}\left(l_{1} \xi^{4}+l_{2} \xi^{3}+l_{3} \xi^{2}+l_{4} \xi+l_{5}\right)=0 \\
k_{1}= & -b_{1}-b_{4}-b_{7}-b_{12}-b_{14} \\
k_{2}= & b_{1} b_{12}+b_{4} b_{12}+b_{7} b_{12}+b_{12} b_{14}+b_{1} b_{4}+b_{1} b_{7}+b_{1} b_{14}+b_{4} b_{7}+b_{4} b_{14} \\
& +b_{7} b_{14}-b_{8} b_{13}+b_{2} b_{6}-b_{5} b_{10} \\
k_{3}= & -b_{1} b_{4} b_{12}-b_{1} b_{7} b_{12}-b_{1} b_{12} b_{14}-b_{4} b_{7} b_{12}-b_{4} b_{12} b_{14}-b_{7} b_{12} b_{14} \\
& -b_{1} b_{4} b_{7}-b_{1} b_{4} b_{14}-b_{1} b_{7} b_{14}-b_{4} b_{7} b_{14} \\
& +b_{1} b_{8} b_{13}+b_{8} b_{12} b_{13}-b_{1} b_{2} b_{6}-b_{2} b_{6} b_{14}-b_{2} b_{6} b_{12}+b_{1} b_{5} b_{10}+b_{5} b_{7} b_{10} \\
& +b_{5} b_{10} b_{14}-b_{2} b_{3} b_{6}+b_{4} b_{8} b_{13}, \\
k_{4}= & b_{1} b_{4} b_{7} b_{12}+b_{1} b_{4} b_{12} b_{14}+b_{1} b_{7} b_{12} b_{14}+b_{4} b_{7} b_{12} b_{14}+b_{1} b_{4} b_{7} b_{14}-b_{1} b_{4} b_{8} b_{13} \\
& -b_{1} b_{12} b_{8} b_{13}-b_{4} b_{8} b_{12} b_{13}+b_{1} b_{2} b_{6} b_{14} \\
& +b_{1} b_{2} b_{6} b_{12}+b_{2} b_{6} b_{12} b_{14}-b_{1} b_{5} b_{7} b_{10}-b_{1} b_{5} b_{10} b_{14}-b_{5} b_{7} b_{10} b_{14} \\
& +b_{5} b_{8} b_{10} b_{13}+b_{2} b_{3} b_{6} b_{14}+b_{2} b_{3} b_{6} b_{12}, \\
k_{5}= & -b_{1} b_{4} b_{7} b_{12} b_{14}+b_{1} b_{4} b_{8} b_{12} b_{13}-b_{1} b_{2} b_{6} b_{12} b_{14}+b_{1} b_{5} b_{7} b_{10} b_{14} \\
& -b_{1} b_{5} b_{8} b_{10} b_{13}-b_{2} b_{3} b_{6} b_{12} b_{14}, \\
l_{1}= & -b_{11}, \\
l_{2}= & b_{1} b_{11}+b_{4} b_{11}+b_{7} b_{11}+b_{11} b_{14}-b_{5} b_{9}, \\
l_{3}= & -b_{1} b_{4} b_{11}-b_{1} b_{7} b_{11}-b_{1} b_{11} b_{14}-b_{4} b_{7} b_{11}-b_{4} b_{11} b_{14}-b_{7} b_{11} b_{14} \\
&
\end{aligned}
$$




$$
\begin{aligned}
& +b_{8} b_{11} b_{13}-b_{2} b_{6} b_{11}+b_{1} b_{5} b_{9}+b_{5} b_{7} b_{9}+b_{5} b_{9} b_{14}, \\
l_{4}= & b_{1} b_{4} b_{7} b_{11}+b_{1} b_{4} b_{11} b_{14}+b_{1} b_{7} b_{11} b_{14}+b_{4} b_{7} b_{11} b_{14}-b_{1} b_{8} b_{11} b_{13} \\
& -b_{4} b_{8} b_{11} b_{13}+b_{1} b_{2} b_{6} b_{11}+b_{2} b_{6} b_{11} b_{14}-b_{1} b_{5} b_{7} b_{9} \\
& -b_{1} b_{5} b_{9} b_{14}-b_{5} b_{7} b_{9} b_{14}+b_{5} b_{8} b_{9} b_{13}+b_{2} b_{3} b_{6} b_{11} \\
l_{5}= & -b_{1} b_{4} b_{7} b_{11} b_{14}+b_{1} b_{4} b_{8} b_{11} b_{13}-b_{1} b_{2} b_{6} b_{11} b_{14}+b_{1} b_{5} b_{7} b_{9} b_{14} \\
& -b_{1} b_{5} b_{8} b_{9} b_{13}-b_{2} b_{3} b_{6} b_{11} b_{14} .
\end{aligned}
$$

Case (i). If $R_{0}>1$ and $\tau=0$.

If $\tau=0$ then equation (4) becomes

$$
\xi^{5}+u_{1} \xi^{4}+u_{2} \xi^{3}+u_{3} \xi^{2}+u_{4} \xi+u_{5}=0
$$

Here, $u_{1}=k_{1}+l_{1}, u_{2}=k_{2}+l_{2}, u_{3}=k_{3}+l_{3}, u_{4}=k_{4}+l_{4}, u_{5}=k_{5}+l_{5}$.

According to the Routh-Hurwitz condition (i) $u_{i}>0, i=1,2,3,4,5$; (ii) $u_{1} u_{2} u_{3}>u_{3}^{2}+$ $u_{1}^{2} u_{4}$; (iii) $\left(u_{1} u_{4}-u_{5}\right)\left(u_{1} u_{2} u_{3}-u_{3}^{2}-u_{1}^{2} u_{4}\right)>u_{5}\left(u_{1} u_{2}-u_{5}\right)^{2}+u_{1} u_{5}^{2}$; hence all the roots of (5) have negative real parts, and therefore the infected steady state $I^{*}$ is locally asymptotically stable.

Case (ii). If $\tau \neq 0$.

If $\tau \neq 0$ then the bifurcation parameter for the delay in immune response when analyzing the existence of Hopf bifurcations starting from infected steady state $I^{*}$.

For $\tau>0$ and $\xi=i p(p>0)$, substituting it in to (4) and separating the real and imaginary parts, we get

$$
\left.\begin{array}{l}
\left(l_{1} p^{4}-l_{3} p^{2}+l_{5}\right) \cos p \tau+\left(-l_{2} p^{3}+l_{4} p\right) \sin p \tau=\left(-k_{1} p^{4}+k_{3} p^{2}-k_{5}\right) \\
\left(-l_{2} p^{3}+l_{4} p\right) \cos p \tau-\left(l_{1} p^{4}-l_{3} p^{2}+l_{5}\right) \sin p \tau=\left(-p^{5}+k_{2} p^{3}-k_{4} p\right)
\end{array}\right\}
$$

It follows from (6) that

$$
p^{10}+c_{1} p^{8}+c_{2} p^{6}+c_{3} p^{4}+c_{4} p^{2}+c_{5}=0
$$

where

$$
\begin{aligned}
& c_{1}=k_{1}^{2}-2 k_{2}-l_{1}^{2}, \quad c_{2}=k_{2}^{2}+2 k_{4}-2 k_{1} k_{3}+2 l_{1} l_{3}-l_{2}^{2}, \\
& c_{3}=k_{3}^{2}+2 k_{1} k_{5}-2 k_{2} k_{4}+2 l_{2} l_{4}-2 l_{1} l_{5}-l_{3}^{2}, \\
& c_{4}=k_{4}^{2}-2 k_{3} k_{5}+2 l_{3} l_{5}-l_{4}^{2}, \quad c_{5}=k_{5}^{2}-l_{5}^{2} .
\end{aligned}
$$

Let $m=p^{2}$, then equation (7) becomes

$$
m^{5}+c_{1} m^{4}+c_{2} m^{3}+c_{3} m^{2}+c_{4} m+c_{5}=0 .
$$

Let $\psi(m)=m^{5}+c_{1} m^{4}+c_{2} m^{3}+c_{3} m^{2}+c_{4} m+c_{5}$, since $\lim _{m \rightarrow \infty} \psi(m)=+\infty$, we conclude that if $c_{5}<0$, then equation (8) has at least one positive root. Suppose that (8) has five positive roots. We have

$$
p_{1}=\sqrt{m_{1}}, \quad p_{2}=\sqrt{m_{2}}, \quad p_{3}=\sqrt{m_{3}}, \quad p_{4}=\sqrt{m_{4}}, \quad p_{5}=\sqrt{m_{5}} .
$$


From (6) we have

$$
\begin{gathered}
\tau_{k}^{(j)}=\frac{1}{p_{k}}\left\{\arccos \left(\frac{a_{1} p_{k}^{8}+a_{2} p_{k}^{6}+a_{3} p_{k}^{4}+a_{4} p_{k}^{2}+a_{5}}{a_{6} p_{k}^{8}+a_{7} p_{k}^{6}+a_{8} p_{k}^{4}+a_{9} p_{k}^{2}+a_{10}}\right)+2 \pi j\right\}, \\
k=1,2,3,4,5, j=0,1,2, \ldots ;
\end{gathered}
$$

where

$$
\begin{aligned}
& a_{1}=l_{2}-k_{1} l_{1}, \quad a_{2}=k_{3} l_{1}+k_{1} l_{3}-k_{2} l_{2}-l_{4}, \\
& a_{3}=k_{4} l_{2}+k_{2} l_{4}-k_{5} l_{1}-k_{3} l_{3}-k_{1} l_{5}, \quad a_{4}=k_{5} l_{3}+k_{3} l_{5}-k_{4} l_{4}, \\
& a_{5}=-k_{5} l_{5}, \quad a_{6}=l_{1}^{2}, \quad a_{7}=l_{2}^{2}-2 l_{1} l_{3}, \quad a_{8}=l_{3}^{2}+2 l_{1} l_{5}-2 l_{2} l_{4}, \\
& a_{9}=l_{4}^{2}-2 l_{3} l_{5}, \quad a_{10}=l_{5}^{2} .
\end{aligned}
$$

$\tau_{0}$ is chosen as $\tau_{0}=\min \left(\tau_{k}^{j}\right)$.We need to prove that $\left[\frac{d(\operatorname{Re} \xi)}{d \tau}\right]_{\tau=\tau_{k}^{(j)}} \neq 0$.

By differentiating equation (4) with respect to $\tau$, we get

$$
\begin{gathered}
\left.\left(\frac{d \xi}{d \tau}\right)=\frac{\xi e^{-\xi \tau}\left(l_{1} \xi^{4}+l_{2} \xi^{3}+l_{3} \xi^{2}+l_{4} \xi+l_{5}\right)}{5 \xi^{4}+4 k_{1} \xi^{3}+3 k_{2} \xi^{2}+2 k_{3} \xi+k_{4}+e^{-\xi \tau}\left[\left(4 l_{1} \xi^{3}+3 l_{2} \xi^{2}+2 l_{3} \xi+l_{4}\right)-\tau\left(l_{1} \xi^{4}+l_{2} \xi^{3}+l_{3} \xi^{2}+l_{4} \xi+l_{5}\right)\right.}\right] \\
\left(\frac{d \xi}{d \tau}\right)^{-1}=\frac{5 \xi^{4}+4 k_{1} \xi^{3}+3 k_{2} \xi^{2}+2 k_{3} \xi+k_{4}}{\xi e^{-\xi \tau}\left(l_{1} \xi^{4}+l_{2} \xi^{3}+l_{3} \xi^{2}+l_{4} \xi+l_{5}\right)} \\
\quad+\frac{4 l_{1} \xi^{3}+3 l_{2} \xi^{2}+2 l_{3} \xi+l_{4}}{\xi\left(l_{1} \xi^{4}+l_{2} \xi^{3}+l_{3} \xi^{2}+l_{4} \xi+l_{5}\right)}-\frac{\tau}{\xi} \\
{\left[\frac{d(\operatorname{Re} \xi)}{d \tau}\right]_{\tau=\tau_{k}^{(j)}}^{-1}=\operatorname{Re}\left[\frac{\left(5 \xi^{4}+4 k_{1} \xi^{3}+3 k_{2} \xi^{2}+2 k_{3} \xi+k_{4}\right) e^{\xi \tau}}{\xi\left(l_{1} \xi^{4}+l_{2} \xi^{3}+l_{3} \xi^{2}+l_{4} \xi+l_{5}\right)}\right]_{\tau=\tau_{k}^{(j)}}} \\
\quad+\operatorname{Re}\left[\frac{4 l_{1} \xi^{3}+3 l_{2} \xi^{2}+2 l_{3} \xi+l_{4}}{\xi\left(l_{1} \xi^{4}+l_{2} \xi^{3}+l_{3} \xi^{2}+l_{4} \xi+l_{5}\right)}\right]_{\tau=\tau}
\end{gathered}
$$

Substituting $\xi=i p$ to be the root of (4), we obtain

$$
\begin{aligned}
= & \frac{1}{\Lambda}\left\{\left[\left(5 p_{k}^{4}-3 k_{2} p_{k}^{2}+k_{4}\right) \cos \left(p_{k} \tau_{k}\right)-\sin \left(p_{k} \tau_{k}\right)\right]\left(l_{2} p_{k}^{4}-l_{4} p_{k}^{2}\right)\right. \\
& +\left[\left(5 p_{k}^{4}-3 k_{2} p_{k}^{2}+k_{4}\right) \sin \left(p_{k} \tau_{k}\right)+\left(-4 k_{1} p_{k}^{3}+2 k_{3} p_{k}\right) \cos \left(p_{k} \tau_{k}\right)\right]\left(l_{1} p_{k}^{5}-l_{3} p_{k}^{3}+l_{5} p_{k}\right) \\
& \left.+\left(-3 l_{2} p_{k}^{2}+l_{4}\right)\left(l_{2} p_{k}^{4}-l_{4} p_{k}^{2}\right)+\left(-4 l_{1} p_{k}^{3}+2 l_{3} p_{k}\right)\left(l_{1} p_{k}^{5}-l_{3} p_{k}^{3}+l_{5} p_{k}\right)\right\},
\end{aligned}
$$

where

$$
\begin{aligned}
\Lambda= & \left(l_{2} p_{k}^{4}-l_{4} p_{k}^{2}\right)^{2}+\left(l_{1} p_{k}^{5}-l_{3} p_{k}^{3}+l_{5} p_{k}^{2}\right) \\
= & \frac{1}{\Lambda}\left\{\left(5 p_{k}^{4}-3 k_{2} p_{k}^{2}+k_{4}\right) p_{k}\left(\left(l_{1} p_{k}^{4}-l_{3} p_{k}^{2}+l_{5}\right) \cos \left(p_{k} \tau_{k}\right)-\left(l_{1} p_{k}^{4}-l_{3} p_{k}^{2}+l_{5}\right) \sin \left(p_{k} \tau_{k}\right)\right)\right. \\
& +\left(-4 k_{1} p_{k}^{3}+2 k_{3} p_{k}\right) p_{k}\left(\left(l_{1} p_{k}^{4}-l_{3} p_{k}^{2}+l_{5}\right) \cos \left(p_{k} \tau_{k}\right)-\left(l_{2} p_{k}^{3}-l_{4} p_{k}\right)\right) \\
& \left.+\left(-3 l_{2} p_{k}^{2}+l_{4}\right)\left(l_{2} p_{k}^{4}-l_{4} p_{k}^{2}\right)+\left(-4 l_{1} p_{k}^{3}+2 l_{3} p_{k}\right)\left(l_{1} p_{k}^{5}-l_{3} p_{k}^{3}+l_{5} p_{k}\right)\right\} \\
= & \frac{1}{\Lambda}\left\{\left(5 p_{k}^{4}-3 k_{2} p_{k}^{2}+k_{4}\right) p_{k}\left(-p_{k}^{5}+k_{2} p_{k}^{3}-k_{4} p_{k}\right)\right.
\end{aligned}
$$




$$
\begin{aligned}
& +\left(-4 k_{1} p_{k}^{3}+2 k_{3} p_{k}\right) p_{k}\left(-k_{1} p_{k}^{4}+k_{3} p_{k}^{2}-k_{5}\right) \\
& \left.+\left(-3 l_{2} p_{k}^{2}+l_{4}\right)\left(l_{2} p_{k}^{4}-l_{4} p_{k}^{2}\right)+\left(-4 l_{1} p_{k}^{3}+2 l_{3} p_{k}\right)\left(l_{1} p_{k}^{5}-l_{3} p_{k}^{3}+l_{5} p_{k}\right)\right\} \\
= & \frac{1}{\Lambda}\left\{5 p_{k}^{10}+4\left(k_{1}^{2}-2 k_{2}-l_{1}^{2}\right) p_{k}^{8}+3\left(k_{2}^{2}+2 k_{4}-2 k_{1} k_{3}+2 l_{1} l_{3}-l_{2}^{2}\right) p_{k}^{6}\right. \\
& \left.+2\left(k_{3}^{2}+2 k_{1} k_{5}-2 k_{2} k_{4}+2 l_{2} l_{4}-2 l_{1} l_{5}-l_{3}^{2}\right) p_{k}^{4}+\left(k_{4}^{2}-2 k_{3} k_{5}+2 l_{3} l_{5}-l_{4}^{2}\right) p_{k}^{2}\right\} \\
= & \frac{1}{\Lambda}\left\{5 p_{k}^{10}+4 c_{1} p_{k}^{8}+3 c_{2} p_{k}^{6}+2 c_{3} p_{k}^{4}+c_{4} p_{k}^{2}\right\} \\
= & \frac{m_{k}}{\Lambda}\left\{5 m_{k}^{4}+4 c_{1} m_{k}^{3}+3 c_{2} m_{k}^{2}+2 c_{3} m_{k}+c_{4}\right\} \\
= & \frac{m_{k}}{\Lambda} \psi^{1}\left(m_{k}\right), \\
\operatorname{sign} & {\left[\frac{d(\operatorname{Re} \xi)}{d \tau}\right]_{\tau=\tau_{k}^{(j)}}=\operatorname{sign}\left[\frac{d(\operatorname{Re} \xi)}{d \tau}\right]_{\tau=\tau_{k}^{(j)}}^{-1}=\operatorname{sign}\left[\frac{\psi^{1}\left(m_{K}\right)}{\Lambda}\right] \neq 0 . }
\end{aligned}
$$

Since $m_{k}, \Lambda>0$ and $\psi^{1}\left(m_{K}\right) \neq 0$.

This completes the theorem.

\section{Estimation of length of delay to preserve stability}

Lemma 4.1 (Nyquist criteria) If $L$ is the arc length of a curve encircling the right half-plane, the curve $\bar{P}_{J}(L)$ will encircle the origin the number of times equal to the difference between the number of poles and the number of zeros of $\bar{P}_{J}(L)$ in the right half-plane.

In this section we shall try to estimate the length of delay to preserve the stability using Nyquist criteria [29-31].

We consider system (2) and the space of all real-valued continuous functions defined on $[-\tau, \infty)$. satisfying the initial conditions on $[-\tau, 0]$.

Let $X(t)=x(t)-x^{*}, Y(t)=y(t)-y^{*}, V(t)=v(t)-v^{*}, W(t)=C(t)-C^{*}, Z(t)=A(t)-A^{*}$.

Linearizing (3) about its steady state $I^{*}$, we obtain

$$
\begin{aligned}
& \dot{X}(t)=\left[-\lambda_{0}+r-\frac{2 r x^{*}}{x_{m}}-\beta_{1} v^{*}\right] X(t)-\beta_{1} x^{*} V(t), \\
& \dot{Y}(t)=\beta_{1} v^{*} X(t)-\left(\delta_{0}+\omega_{1} C^{*}\right) Y(t)+\beta_{1} x^{*} V(t)-\omega_{1} y^{*} W(t), \\
& \dot{V}(t)=N_{1} \delta_{0} Y(t)-\left(\delta_{1}+\omega_{2} A^{*}\right) V(t)-\omega_{2} v^{*} Z(t), \\
& \dot{W}(t)=\alpha_{1} Y(t)-\mu_{10} W(t)+\mu_{1} C^{*} Y(t-\tau)+\mu_{1} y^{*} W(t-\tau), \\
& \dot{Z}(t)=\mu_{2} A^{*} V(t)+\left(\mu_{2} v^{*}-\mu_{20}\right) Z(t) .
\end{aligned}
$$

Let $P_{1}=\left(-\lambda_{0}+r-\frac{2 r x^{*}}{x_{m}}-\beta_{1} v^{*}\right), P_{2}=-\beta_{1} x^{*}$,

$$
\begin{aligned}
& Q_{1}=\beta_{1} v^{*}, \quad Q_{2}=-\left(\delta_{0}+\omega_{1} C^{*}\right), \quad Q_{3}=\beta_{1} x^{*}, \quad Q_{4}=-\omega_{1} y^{*}, \\
& R_{1}=N_{1} \delta_{0}, \quad R_{2}=-\left(\delta_{1}+\omega_{2} A^{*}\right), \quad R_{3}=-\omega_{2} v^{*}, \\
& T_{1}=\alpha_{1}, \quad T_{2}=-\mu_{10}, \quad T_{3}=\mu_{1} C^{*}, \quad T_{4}=\mu_{1} y^{*}, \\
& U_{1}=\mu_{2} A^{*}, \quad U_{2}=\left(\mu_{2} v^{*}-\mu_{20}\right) .
\end{aligned}
$$


Equation (12) becomes

$$
\begin{aligned}
& \dot{X}(t)=P_{1} X(t)+P_{2} V(t), \\
& \dot{Y}(t)=Q_{1} X(t)+Q_{2} Y(t)+Q_{3} V(t)+Q_{4} W(t), \\
& \dot{V}(t)=R_{1} Y(t)+R_{2} V(t)+R_{3} Z(t), \\
& \dot{W}(t)=T_{1} Y(t)+T_{2} W(t)+T_{3} Y(t-\tau)+T_{4} W(t-\tau), \\
& \dot{Z}(t)=U_{1} V(t)+U_{2} Z(t) .
\end{aligned}
$$

Taking the Laplace transform on both sides of (13), we obtain

$$
\begin{aligned}
& s L[X(t)]-X(0)=P_{1} L[X(t)]+P_{2} L[V(t)], \\
& L[Y(t)]-Y(0)=Q_{1} L[X(t)]+Q_{2} L[Y(t)]+Q_{3} L[V(t)]+Q_{4} L[W(t)], \\
& s L[V(t)]-V(0)=R_{1} L[Y(t)]+R_{2} L[V(t)]+R_{3} L[Z(t)], \\
& s L[W(t)]-W(0)=T_{1} L[Y(t)]+T_{2} L[W(t)]+T_{3} e^{-s \tau} L[Y(t)]+T_{3} e^{-s \tau} K_{1}(s) \\
& +T_{4} e^{-s \tau} L[W(t)]+T_{4} e^{-s \tau} K_{2}(s), \\
& s L[Z(t)]-Z(0)=U_{1} L[V(t)]+U_{2} L[Z(t)] \text {, }
\end{aligned}
$$

where $K_{1}(s)=\int_{-\tau}^{0} e^{-s t} Y(t) d t, K_{2}(s)=\int_{-\tau}^{0} e^{-s t} W(t) d t$.

The local asymptotic stability of the infected steady state $I^{*}$ according to the Nyquist criterion is given by

$$
\begin{aligned}
& \operatorname{Im} H\left(i \rho_{0}\right)>0, \\
& \operatorname{Re} H\left(i \rho_{0}\right)=0,
\end{aligned}
$$

where $\rho_{0}$ is the smallest positive root of (15) and

$$
H(s)=s^{5}+k_{1} s^{4}+k_{2} s^{3}+k_{3} s^{2}+k_{4} s+k_{5}+e^{-s \tau}\left(l_{1} s^{4}+l_{2} s^{3}+l_{3} s^{2}+l_{4} s+l_{5}\right) .
$$

From (14) and (15) we have

$$
\begin{aligned}
& k_{1} \rho_{0}^{4}-k_{3} \rho_{0}^{2}+k_{5}=-\left(l_{1} \rho_{0}^{4}-l_{3} \rho_{0}^{2}+l_{5}\right) \cos \left(\rho_{0} \tau\right)-\left(-l_{2} \rho_{0}^{3}+l_{4} \rho_{0}\right) \sin \left(\rho_{0} \tau\right), \\
& \rho_{0}^{5}-k_{2} \rho_{0}^{3}+k_{4} \rho_{0}>\left(l_{1} \rho_{0}^{4}-l_{3} \rho_{0}^{2}+l_{5}\right) \sin \left(\rho_{0} \tau\right)-\left(-l_{2} \rho_{0}^{3}+l_{4} \rho_{0}\right) \cos \left(\rho_{0} \tau\right) .
\end{aligned}
$$

Conditions (16) and (17) are the smallest conditions to ensure stability. We will use these to obtain an estimate of the length of delay. Our goal is to find an upper bound $\rho_{+}$on $\rho_{0}$, independent of $\tau$, and we estimate $\tau$ equation (17) holds for all values of $\rho_{0}, 0<\rho_{0}<\rho_{+}$. Maximizing the R.H.S of (16)

Subject to $\left|\cos \left(\rho_{0} \tau\right)\right| \leq 1$ and $\left|\sin \left(\rho_{0} \tau\right)\right| \leq 1$, we obtain

$$
k_{1} \rho_{0}^{4} \leq k_{3} \rho_{0}^{2}-k_{5}+\left|l_{1}\right| \rho_{0}^{4}+\left|l_{3}\right| \rho_{0}^{2}+\left|l_{5}\right|+\left|l_{2}\right| \rho_{0}^{3}+\left|l_{4}\right| \rho_{0} .
$$

It is clear that $\rho_{0} \leq \rho_{+}$, where $\rho_{+}$is the least positive root of (18). 
From (17) we have

$$
\begin{aligned}
\rho_{0}^{4}> & k_{2} \rho_{0}^{2}-k_{4}+l_{1} \rho_{0}^{3} \sin \left(\rho_{0} \tau\right)-l_{3} \rho_{0} \sin \left(\rho_{0} \tau\right)+l_{5} \frac{\sin \left(\rho_{0} \tau\right)}{\rho_{0}} \\
& +l_{1} \rho_{0}^{2} \cos \left(\rho_{0} \tau\right)-l_{4} \cos \left(\rho_{0} \tau\right)
\end{aligned}
$$

Substituting (16) into (19) yields

$$
\begin{aligned}
& \left(k_{1} l_{1} \rho_{0}^{2}-k_{1} l_{4}+l_{1} \rho_{0}^{4}-l_{3} \rho_{0}^{2}+l_{5}\right)\left(\cos \left(\rho_{0} \tau\right)-1\right) \\
& \quad+\left(k_{1} l_{1} \rho_{0}^{3}-k_{1} l_{3} \rho_{0}+\frac{k_{1} l_{5}}{\rho_{0}}-l_{2} \rho_{0}^{3}+l_{4} \rho_{0}\right) \sin \left(\rho_{0} \tau\right) \\
& \quad<\left(-l_{1}\right) \rho_{0}^{3}+\left(k_{3}-k_{1} k_{2}-k_{1} l_{1}+l_{3}\right) \rho_{0}^{2}+\left(k_{1} k_{4}-k_{5}-l_{5}+k_{1} l_{4}\right) .
\end{aligned}
$$

By using the bounds

(i) $\left(k_{1} l_{1} \rho_{0}^{2}-k_{1} l_{4}+l_{1} \rho_{0}^{4}-l_{3} \rho_{0}^{2}+l_{5}\right)\left(\cos \left(\rho_{0} \tau\right)-1\right)$

$$
\begin{aligned}
& =\left(k_{1} l_{1} \rho_{0}^{2}-k_{1} l_{4}+l_{1} \rho_{0}^{4}-l_{3} \rho_{0}^{2}+l_{5}\right) 2 \sin ^{2}\left(\frac{\rho_{0} \tau}{2}\right) \\
& <2\left(k_{1} l_{1} \rho_{0}^{2}-k_{1} l_{4}+l_{1} \rho_{0}^{4}-l_{3} \rho_{0}^{2}+l_{5}\right)\left(\frac{\rho_{0} \tau}{2}\right)^{2} \\
& <\frac{1}{2}\left(l_{1} \rho_{0}^{6}+\left(k_{1} l_{1}-l_{3}\right) \rho_{0}^{4}+\left(l_{5}-k_{1} l_{4}\right) \rho_{0}^{2}\right) \tau^{2} .
\end{aligned}
$$

(ii) $\left(k_{1} l_{1} \rho_{0}^{3}-k_{1} l_{3} \rho_{0}+\frac{k_{1} l_{5}}{\rho_{0}}-l_{2} \rho_{0}^{3}+l_{4} \rho_{0}\right) \sin \left(\rho_{0} \tau\right)$

$$
\begin{aligned}
& <\left(k_{1} l_{1} \rho_{0}^{3}-k_{1} l_{3} \rho_{0}+\frac{k_{1} l_{5}}{\rho_{0}}-l_{2} \rho_{0}^{3}+l_{4} \rho_{0}\right) \rho_{0} \tau \\
& <\left(\left(k_{1} l_{1}-l_{2}\right) \rho_{0}^{4}+\left(l_{4}-k_{1} l_{3}\right) \rho_{0}^{2}+k_{1} l_{5}\right) \tau .
\end{aligned}
$$

In (20) we obtain $B_{1} \tau^{2}+B_{2} \tau<B_{3}$, where

$$
\begin{aligned}
& B_{1}=\frac{1}{2}\left(l_{1} \rho_{0}^{6}+\left(k_{1} l_{1}-l_{3}\right) \rho_{0}^{4}+\left(l_{5}-k_{1} l_{4}\right) \rho_{0}^{2}\right), \\
& B_{2}=\left(k_{1} l_{1}-l_{2}\right) \rho_{0}^{4}+\left(l_{4}-k_{1} l_{3}\right) \rho_{0}^{2}+k_{1} l_{5}, \\
& B_{3}=-l_{1} \rho_{0}^{3}+\left(k_{3}-k_{1} k_{2}-k_{1} l_{1}+l_{3}\right) \rho_{0}^{2}+\left(k_{1} k_{4}-k_{5}-l_{5}+k_{1} l_{4}\right) .
\end{aligned}
$$

Hence if $\tau_{+}=\frac{-B_{2}+\sqrt{B_{2}^{2}+4 B_{1} B_{3}}}{2 B_{1}}$, then the stability is preserved for $0<\tau<\tau_{+}$. Therefore, we get Theorem 4.1 .

Theorem 4.1 If there exists a delay in $0<\tau<\tau_{+}$such that $B_{1} \tau^{2}+B_{2} \tau<B_{3}$, then $\tau_{+}$is locally asymptotically stable, the delay length for the infected steady state $I^{*}$.

\section{Results and discussion}

We exhibit some numerical assumptions with respect to our hypothetical investigation which are done in MATLAB. Using the following parameter values

$$
\lambda=10, \quad \lambda_{0}=0.055, \quad \delta_{0}^{1}=0.24, \quad \delta_{1}=3, \quad \beta=0.002, \quad \alpha_{0}=0.265,
$$




$$
\begin{array}{ll}
\alpha_{1}^{1}=0.01, \quad \mu_{10}=0.755, \quad \mu_{20}=0.1, \quad \mu_{1}=0.03, \quad \mu_{2}=0.01, \quad \eta_{r}=0.57, \\
\eta_{p}=0.38, \quad \omega_{1}=0.05, \quad \omega_{2}=0.5, \quad N=100, \quad x_{m}=1500, \quad r=0.3,
\end{array}
$$

we get the infected steady state $I^{*}\left(x^{*}, y^{*}, v^{*}, C^{*}, A^{*}\right)=(1222.89,21.8376,10,4.4869$, 63.6732). From (8) and (9) we obtain $\tau_{0}=1.102$ and $R_{0}=21.7348>1$. In case that the delay moderately increases from zero, at that point the infected steady state $I^{*}$ is locally asymptotically stable for $\tau \in\left[0, \tau_{0}\right.$ ) (by Theorem 3.1) which is outlined in Fig. 1 . In any case, the delay crosses the critical value $\tau_{0}=1.102$, the infected steady state $I^{*}$ loses stability and undergoes Hopf bifurcation, which is illustrated in Fig. 2. The proposed demonstration suggests that as the response of antibody often evolves as possible in free virus
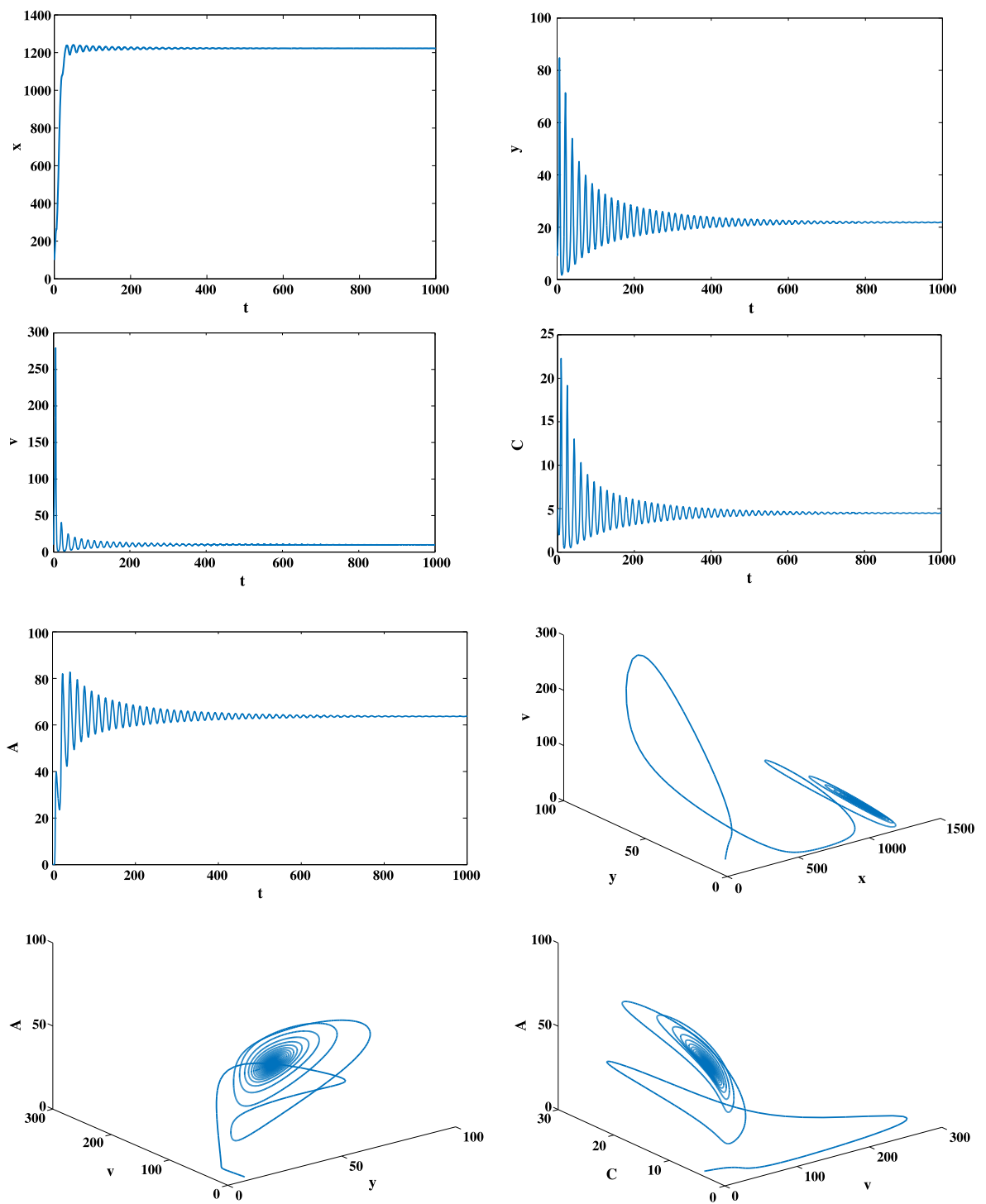

Figure 1 The phase diagrams of system (2) being asymptotically stable when $\tau=0.9$ 

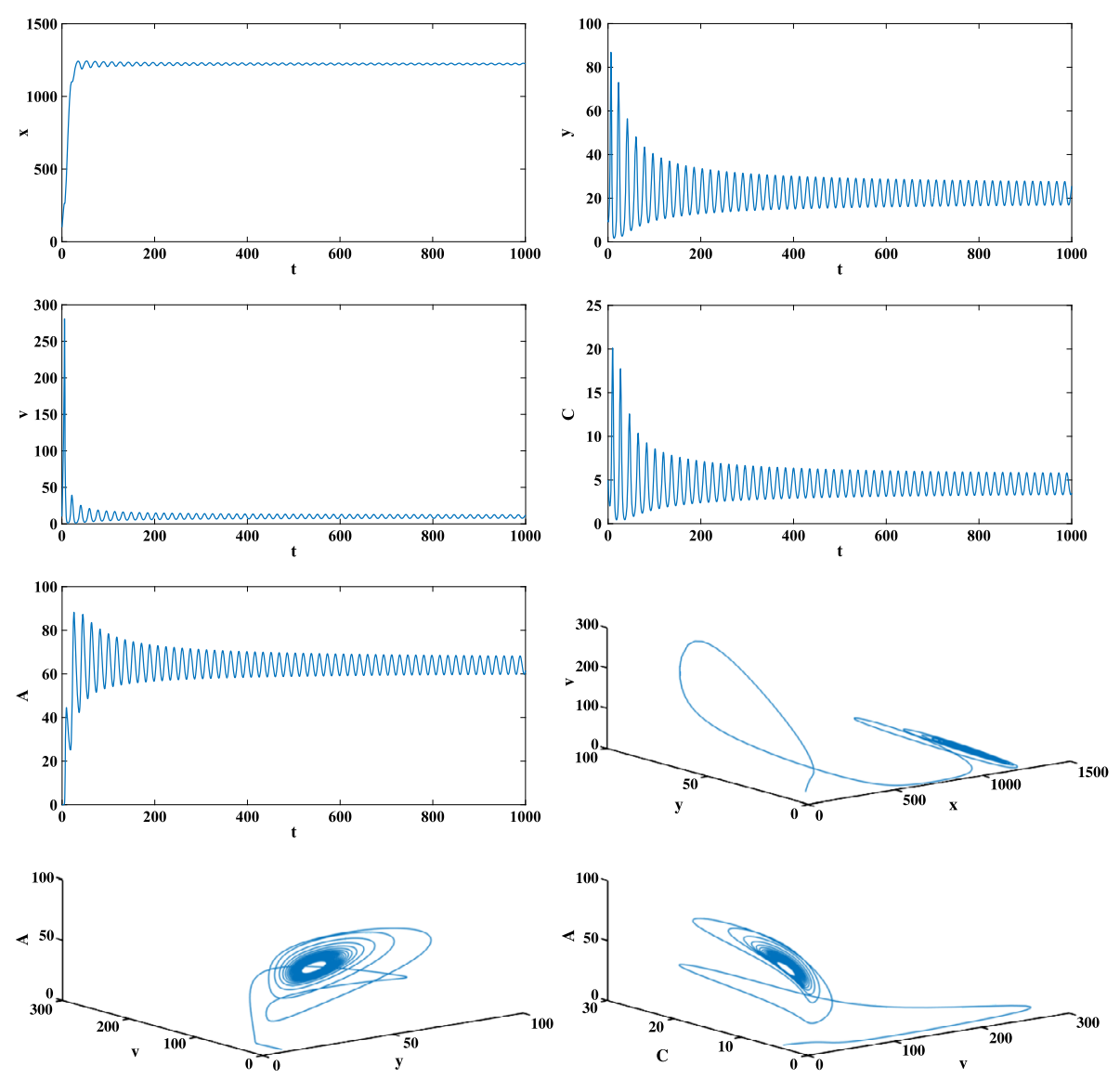

Figure 2 The phase diagrams of system (2) undergoing Hopf bifurcation when $\tau=1.2$

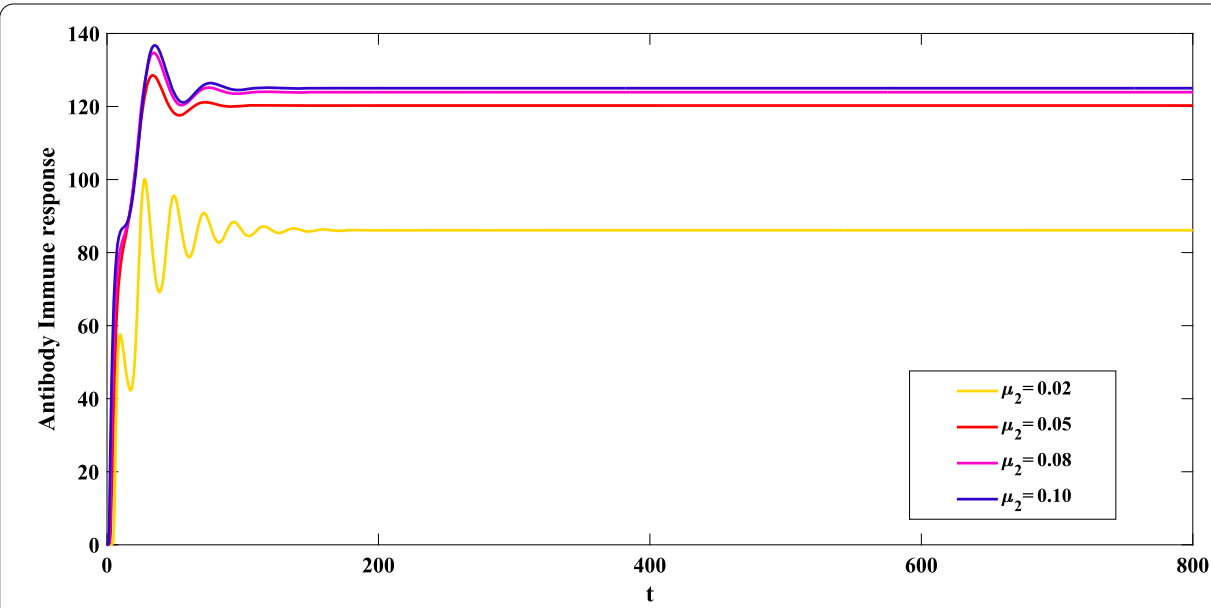

Figure 3 The phase diagram shows that the neutralizing antibody converges to a certain extent after the regulation of free viruses and infected cells

response, then the level of non-infected $\mathrm{CD} 4^{+} \mathrm{T}$-cells increases with a time delay as shown in Figs. 3 and 4.In the meantime, the level of infected CD $4^{+}$T-cells and free virus decreases as shown in Figs. 5 and 6. 


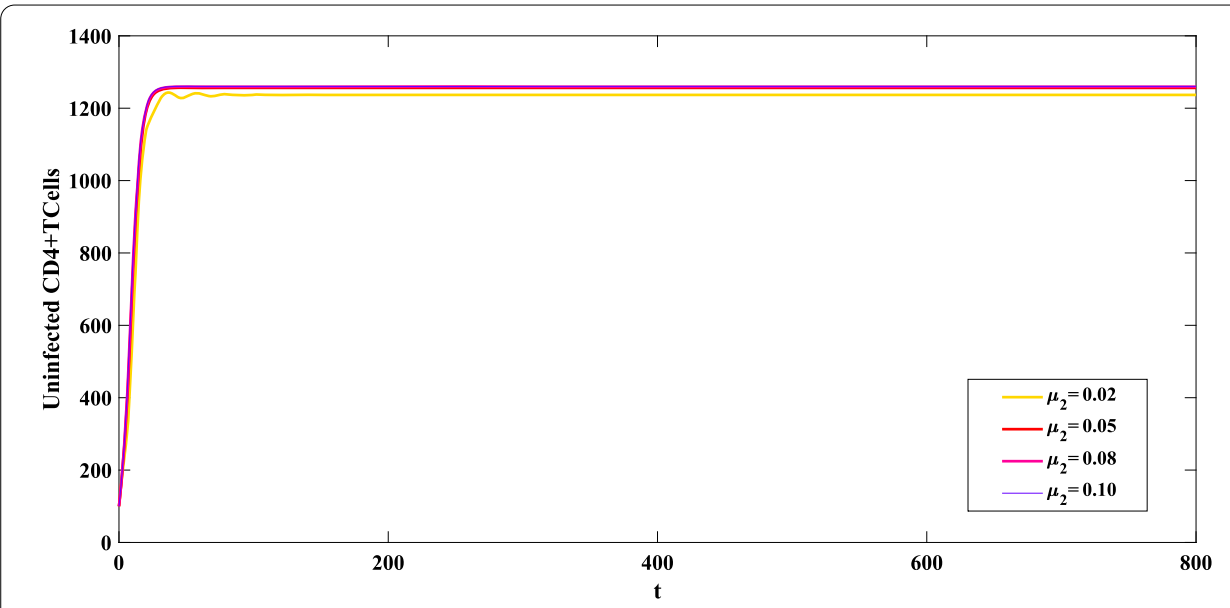

Figure 4 The phase diagram shows an increased number of non-infected CD4 ${ }^{+}$T-cells, while the antibody neutralization response is increasing

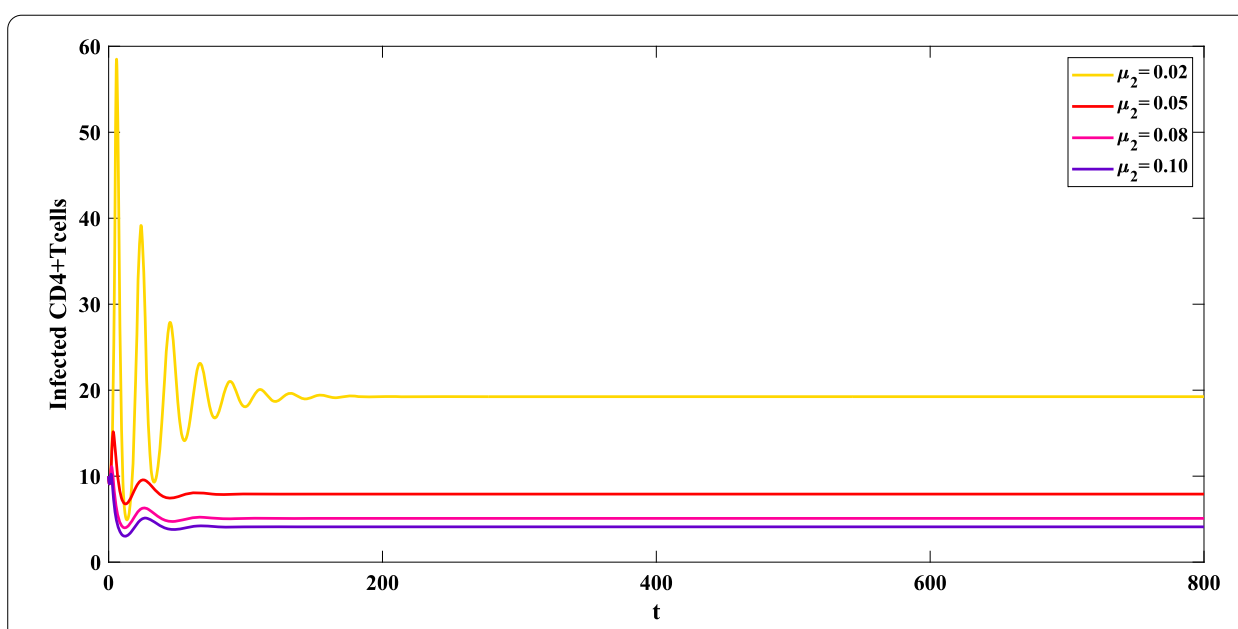

Figure 5 The phase diagram demonstrates that the neutralization of the antibody regulates the infected cells as antibody neutralization increases

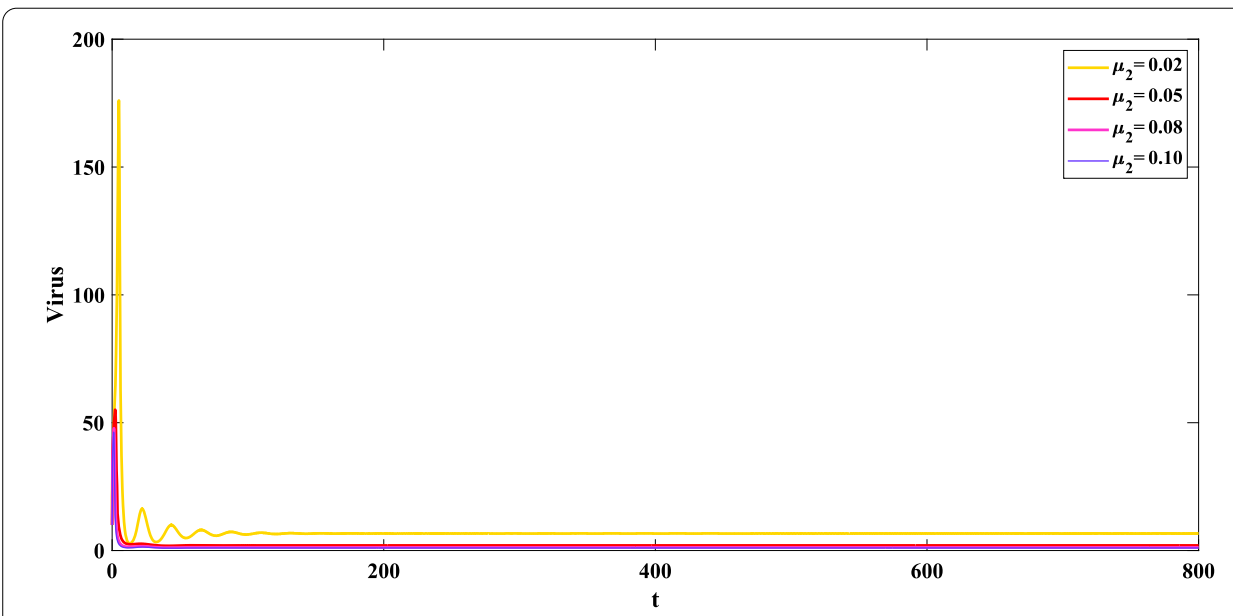

Figure 6 The phase diagram shows that when neutralization of antibody response increases and the free virus level converges to zero, the free virus is neutralized 


\title{
6 Conclusion
}

In this study, the dynamics of an HIV infection model were analyzed and described by comprising the CTL response delay according to mathematical analysis. We have shown that the reproduction ratio $R_{0}$ plays a crucial role for inferring this model dynamics. The calculation of $R_{0}$ in an infectious disease transmitted by blood or sex is more complicated. Several mathematical models have been proposed to explain the rate of spread of the infection. An account should be taken of the window period and HIV pathophysiology. Better integration of modeling into the decision-making process is desirable to improve the effectiveness of interventions and the knowledge of health professionals [32].

Both the viral free steady state and the infected steady state of the HIV model are ascertained. If $R_{0}<1$ then the viral free steady state is locally asymptotically stable, and if $R_{0}>1$ then infected steady state is locally asymptotically stable in absence of delay. Based on the model of differential delay, we evaluate Hopf bifurcation requirements using time delay as the bifurcation parameter. This shows that a positive steady state is locally asymptotically stable when the time delay is relatively small, whereas a Hopf bifurcation may cause a loss of stability as the delay increases. Using the Nyquist test, we also get that the maximum delay value to the infected steady State $I^{*}$ will remain asymptomatically stable. Finally, it can be concluded that an antibody immune reaction and the combination of drug efficacy are important to reduce the potential for HIV infections through numerical simulations. Maintaining stability may be the goal to prolonging patient survival, but exposes them to transmission risk. It is interesting to know the duration of the asymptotic period for subsequent consequent clinical evaluations. Dynamic HIV transmission models can provide evidence-based guidance on optimal combination implementation strategies to treat and prevent HIV/AIDS [33].

\begin{abstract}
Acknowledgements
The authors wish to thank the chief editor, the associate editor, and the anonymous reviewers for their helpful feedback to improve the quality of this manuscript. The authors also wish to thank Dr. R. Ponalagusamy, Professor, Department of Mathematics, National Institute of Technology, Trichy, India, and Dr. V. Vetrivel, Professor, Department of Mathematics, Indian Institute of Technology Madras, Chennai, India, for their valuable guidance. We also thankful to Dr. K. Kamalanand, Assistant Professor, Department of Instrumentation Engineering, Madras Institute of Technology Campus, Anna University, Chromepet, Chennai, for his valuable suggestion.

Funding

The financial support provided in the form of a University Research Association (URF) by SRM Institute of Science and Technology, Chennai, is thankfully recognized.
\end{abstract}

Availability of data and materials

The analysis in this article did not generate data.

Competing interests

The authors declare that there are no competing interests.

Authors' contributions

The authors were equally involved in writing this paper and read the final copy and agreed to the manuscript.

Author details

${ }^{1}$ Department of Mathematics, SRM Institute of Science and Technology, Tamil Nadu, India. ${ }^{2}$ Department of Mathematics, Faculty of Engineering and Technology, SRM Institute of Science and Technology, Kattankulathur-603 203, Chengalpattu District, Tamil Nadu, India.

\section{Publisher's Note}

Springer Nature remains neutral with regard to jurisdictional claims in published maps and institutional affiliations. 


\section{References}

1. Kirschner, D.: Using mathematics to understand HIV immune dynamics. Not. Am. Math. Soc. 43, 191-202 (1996)

2. Bonhoeffer, S., May, R.M., Shaw, G.M., Nowak, M.A.: Virus dynamics and drug therapy. Proc. Natl. Acad. Sci. 94, 6971-6976 (1997)

3. Perelson, A.S., Nelson, P.W.: Mathematical analysis of HIV-I dynamics in vivo. SIAM Rev. 41, 3-44 (1999)

4. Srivastava, P.K., Banerjee, M., Chandra, P.: Dynamical model of in-host HIV infection: with drug therapy and multi viral strains. J. Biol. Syst. 20, 303-325 (2012)

5. Dorratoltaj, N., Nikin-Beers, R., Ciupe, S.M., Eubank, S.G., Abbas, K.M.: Multi-scale immunoepidemiologic modeling of within-host and between-host HIV dynamics: systematic review of mathematical models. PeerJ 5, e3877 (2017)

6. Nishiura, H.: Estimating the incidence and diagnosed proportion of HIV infections in Japan: a statistical modeling study. PeerJ 7, e6275 (2019)

7. Wodarz, D., Nowak, M.A.: Mathematical models of HIV pathogenesis and treatment. BioEssays 24, 1178-1187 (2002)

8. Ashrafur Rahman, S.M., Vaidya, N.K., Zou, X.: Impact of early treatment programs on HIV epidemics: an immunity-based mathematical model. Math. Biosci. 280, 38-49 (2016)

9. Wang, L., Li, M.Y.: Mathematical analysis of the global dynamics of a model for HIV infection of CD4 ${ }^{+} \mathrm{T}$ cells. Math. Biosci. 200, 44-57 (2006)

10. Wodarz, D., Krakauer, D.C.: Defining CTL-induced pathology: implications for HIV. Virology 274, 94-104 (2000)

11. Nowak, M.A., Bangham, C.R.M.: Population dynamics of immune responses to persistent viruses. Science 272, 74-79 (1996)

12. Wang, X., Elaiw, A., Song, X.: Global properties of a delayed HIV infection model with CTL immune response. Appl. Math. Comput. 218, 9405-9414 (2012)

13. Chan, B.S., Yu, P.: Bifurcation analysis in a model of cytotoxic T-lymphocyte response to viral infections. Nonlinear Anal., Real World Appl. 13, 64-77 (2012)

14. Miao, H., Teng, Z.. Abdurahman, X.: Stability and Hopf bifurcation for five-dimensional virus infection model with Beddington-DeAngelis incidence and three delays. J. Biol. Dyn. 12, 146-170 (2018)

15. Meskaf, A., Tabit, Y., Allali, K.: Global analysis of a HCV model with CTL, antibody responses and therapy. Appl. Math. Sci. 9, 3997-4008 (2015)

16. Wodarz, D.: Hepatitis C virus dynamics and pathology: the role of CTL and antibody responses. J. Gen. Virol. 84, $1743-1750$ (2003)

17. Yousfi, N., Hattaf, K., Rachik, M.: Analysis of a HCV model with CTL and antibody responses. Appl. Math. Sci. 3, 2835-2846 (2009)

18. Yan, Y., Wang, W.: Global stability of a five-dimensional model with immune response and delay. Discrete Contin. Dyn. Syst., Ser. B 17, 401-416 (2012)

19. Culshaw, R.V., Ruan, S., Webb, G.: A mathematical model of cell-to-cell spread of HIV-1that includes a time delay. J. Math. Biol. 46, 425-444 (2003)

20. Li, M.Y., Shu, H.: Global dynamics of an in-host viral model with intracellular delay. Bull. Math. Biol. 72, 1492-1505 (2010)

21. Xu, R.: Global stability of an HIV-1 infection model with saturation infection and intracellular delay. J. Math. Anal. Appl. 375, 75-81 (2011)

22. Zhu, H., Luo, Y., Chen, M.: Stability and Hopf bifurcation of a HIV infection model with CTL-response delay. Comput. Math. Appl. 62, 3091-3102 (2011)

23. El Boukari, B., Yousfi, N.: A delay differential equation model of HIV infection, with therapy and CTL response. Bull. Math. Sci. Appl. 9, 53-68 (2014)

24. Sahani, S.K., Yashi: A delayed HIV infection model with apoptosis and viral loss. J. Biol. Dyn. 12, 1012-1034 (2018)

25. Dubey, P., Dubey, U.S., Dubey, B.: Modeling the role of acquired immune response and antiretroviral therapy in the dynamics of HIV infection. Math. Comput. Simul. 144, 120-137 (2018)

26. Hale, J.: Theory of Functional Differential Equations. Springer, New York (1997)

27. Kuang, Y:: Delay Differential Equations with Applications in Population Dynamics. Academic Press, San Diego (1993)

28. Miao, H., Kang, C.: Stability and Hopf bifurcation analysis for an HIV infection model with Beddington-DeAngelis incidence and two delays. J. Appl. Math. Comput. 60, 265-290 (2019)

29. Erbe, L.H., Freedman, H.I., Rao, V.S.: Three-species food chain models with mutual interference and time delays. Math. Biosci. 80, 57-80 (1986)

30. Jiang, X., Zhou, X., Shi, X., Song, X.: Analysis of stability and Hopf bifurcation for a delay differential equation model of HIV infection of CD4 ${ }^{+}$T-cells. Chaos Solitons Fractals 38, 447-460 (2008)

31. LV, Y., Hu, Z., Liao, F.: The stability and Hopf bifurcation for an HIV model with saturated infection rate and double delays. Int. J. Biomath. 11, 1850040-1850043 (2018)

32. Marranzano, M., Ragusa, R., Platania, M., Faro, G., Coniglio, M.: Knowledge, attitudes and practices towards patients with HIV/AIDS in staff nurses in one university hospital in Sicily. Epidemiol. Biostat. Public Health 10, e8731 (2013)

33. Krebs, E., Enns, B., Wang, L., et al.: Localized HIV modelling study group. Developing a dynamic HIV transmission model for 6 U.S. cities: an evidence synthesis. PLoS ONE 14(5), e0217559 (2019) 